\title{
A Comparison of GPS Workload Demands in Match Play and Small-Sided Games by the Positional Role in Youth Soccer
}

\author{
by \\ Mathew Beenham ${ }^{1}$, David J. Barron ${ }^{1}$, John Fry ${ }^{1}$, Howard H. Hurst ${ }^{2}$, \\ Antonio Figueirdo², Steve Atkins ${ }^{2}$
}

The external demands of small-sided games (SSGs) according to the positional role are currently unknown. Using a Catapult Minimax X3 $5 \mathrm{~Hz}$ GPS, with a $100 \mathrm{~Hz}$ tri-axial accelerometer, we compared the accumulated triaxial player workload per min (PLacc.min $\left.{ }^{-1}\right)$ during friendly youth match play (MP) (11 vs. 11) and SSGs (2 vs. 2, 3 vs. 3, and 4 vs. 4). Significant differences existed between all SSGs and MP for PLacc. min $^{-1}(F=21.91, p<0.001, \eta 2=$ $0.38)$, and individual $X(F=27.40, p<0.001, \eta 2=0.43), Y(F=14.50, p<0.001, \eta 2=0.29)$ and $Z(F=19.28, p<$ $0.001, \eta 2=0.35)$ axis loads. Across all conditions, mean PLacc. min ${ }^{-1}$ was greater for midfielders ( $p=0.004$, CI: 0.68 , $4.56)$ and forwards $(p=0.037, C I: 0.08,3.97)$ than central defenders. In all conditions, greater $Y$ axis values existed for wide defenders ( $p=0.024, C I: 0.67,1.38)$, midfielders ( $p=0.006, C I: 0.18,1.50)$ and forwards $(p=0.007, C I: 0.17$, 0.15) compared to central defenders. Midfielders reported greater $Z$ axis values compared to central defenders ( $p=$ 0.002, CI: 0.40, 2.23). We concluded that SSGs elicited greater external loads than MP, and previous studies may have underestimated the demands of SSGs.

Key words: small sided games, training games, conditioning, soccer, player load, GPS.

\section{Introduction}

Small sided games (SSGs) are widely used to simultaneously improve fitness, tactical awareness, and specific dynamics of the game (Hill-Haas et al., 2011). Through the manipulation of the number of players, pitch dimensions, work and rest periods, rules of the game, coach encouragement, and inclusion of goalkeepers, different physical, technical and tactical responses may be elicited (Dellal et al., 2008; Little and Williams, 2006, 2007; Mallo and Navarro, 2008; Rampinini et al., 2007). Increasing pitch dimensions, while maintaining a constant number of players, leads to an increased mean heart rate, blood lactate concentration and rating of perceived exertion (Clemente et al., 2014). In contrast, small pitch dimensions increase the technical demands, evidenced by a higher frequency of tackles and shots (Kelly and Drust, 2009). Although these studies provided a greater understanding of the rigours of SSG, internal physiological measures are not the most appropriate for the determination of physical demands. This has been aligned with sensitivity issues and, as a result, an underestimation of the internal demands of the game. For example, the heart rate has been found to respond slowly to changes in exercise intensity and is, therefore, an inappropriate measuring tool (Achten and Jeukendrup, 2003; Borresen and Lambert, 2008).

Alternatively, Global Positioning Systems (GPSs) have been widely adopted to evalute physical performance (Edgecomb and Norton 2006; Gabbett, 2010; Petersen et al., 2011). These devices enable temporal and kinematic variables

1 - Centre for Research in Sports Performance, Myerscough College, UK.

2 - Centre for Applied Sport and Exercise Sciences, University of Central Lancashire, UK. 
such as distance, direction of movement and velocity to be measured (Scott et al., 2013). Using GPSs in game situations has revealed that SSGs played on small pitch dimensions evoke the majority of the features occurring in match play (MP), but are insufficient to reproduce the highintensity and repeated-sprint demands of highlevel competition (Casamichana et al., 2012; Gabbett and Mulvey, 2008). However, SSGs played on larger pitch dimensions stimulate significantly greater high-speed running than MP (Dellal et al., 2012). To assess the physical demands of soccer, much attention is paid to activities completed at high-intensity (Bradley et al., 2010; Mohr et al., 2003). However, this approach is flawed because it fails to account for low-speed movements that are energetically costly and are a key feature of match play. For example, accelerations and decelerations, jumps, turns and physical contacts may be classified into low-speed activity, despite evoking high physiological loads (Osgnach et al., 2010; Reilly and Bowen, 1984; Varley and Aughey, 2013). Despite recent improvements in sampling frequency, GPSs remain insensitive to some discrete movements and inaccuracies are found when measuring high-speed activities and rapid changes of direction (Jennings et al., 2010; Rawstorn et al., 2014). Interestingly, increased sampling frequency may not improve sensitivity during soccer activity because when compared to $10 \mathrm{~Hz}$ GPS, $15 \mathrm{~Hz}$ models demonstrated inferior validity and interunit reliability (Johnston et al., 2014). Furthermore, measurements of acceleration and deceleration exhibit greater inter-unit variability (Buchheit et al., 2014) leading the same authors to conclude that care should be taken when comparing data with different models and/or units.

Using GPS units with integrated tri-axial accelerometers enables the measurement of the total mechanical stress associated with discrete game activities. Importantly, accelerations are more energetically demanding than movement at a constant velocity (Osgnach et al., 2010) and their omission would underestimate the rigour of competition. Furthermore, decelerations induce significant mechanical stress on the body, explained by the associated eccentric muscular action (Thompson et al., 1999), and occur as frequently as accelerations (Osgnach et al., 2016).
These systems avoid some of the limitations previously outlined with internal measures and time-motion analysis, as they typically provide a sampling rate of $100 \mathrm{~Hz}$ and report excellent accuracy and reliability (CV $1.02-1.04 \%$ ) (Boyd et al., 2011).

The summation of acceleration and deceleration movements in each anatomical plane (X: medial-lateral, Y: anterior-posterior, Z: caudalcranial) provides an estimate of the total player workload (PL) (Boyd et al., 2011; Cummins et al., 2013). The application of PL has differentiated between SSGs and competition demands of basketball (Montgomery et al., 2010) as well as between playing positions in basketball, netball and soccer (Barron et al., 2014; Cormack et al., 2013; Montgomery et al., 2010). The player workload correlates with internal measures including HR-based and rating of perceived exertion (RPE)-based measures $(\mathrm{r}=0.71-0.84)$, and total distance covered $(r=0.93)$ (Scott et al., 2013).

With regard to soccer, research has reported PL values for SSGs and MP (Aguiar et al., 2013; Casamichana et al., 2012). However, these studies provided accumulated PL values (PLacc) rather than the individual contribution of each anatomical plane. To the best of our knowledge, to date only Barron et al. (2014) have reported the tri-axial load during competitive youth soccer, highlighting significant differences in the $\mathrm{Y}$ axis load between centre midfielders and central defenders.

Further information regarding the contribution of each individual plane to PLacc would provide a greater understanding of the discreet movements performed by players of different positional roles. Therefore, the aims of this study were to determine the tri-axial external load of three SSG formats (2 vs. 2, 3 vs. 3 and 4 vs. 4) and secondly, through comparison with data derived during 11 vs. 11 competition, to understand how each SSG format reflected the triaxial activity of competition.

\section{Material and Methods}

\section{Participants}

Forty trained high level youth soccer players (age $17.0 \pm 0.6$ yrs, body mass $73.93 \pm 5.85$ $\mathrm{kg}$, stature $180 \pm 6 \mathrm{~cm}$ ) volunteered for the study and provided parental consent in accordance with the Declaration of Helsinki. Players were 
classified by the playing position, including central defenders $(C D=10)$, wide defenders (WD $=10)$, midfielders $(\mathrm{MF}=10)$ and forwards $(\mathrm{FW}=$ 10). Wide midfielders were not included in the study as a result of the playing formation used. Approval for the study was granted by the University of Central Lancashire Ethics Committee.

Equipment

External loads were determined using a GPS sampling at $5 \mathrm{~Hz}$, which included a tri-axial accelerometer sampling at $100 \mathrm{~Hz}$ (Minimax X3, Catapult Innovations, Australia). Devices were secured between the upper scapulae, at approximately the T3-4 junction. The devices were activated 15 minutes before use, in accordance with the manufacturer's instructions, and to allow satellites to download the required almanac data. Player workload values are presented as the individual $\mathrm{X}, \mathrm{Y}$ and $\mathrm{Z}$ anatomical plane components and arbitrary units (AU). To avoid bias due to the different duration of SSGs and MP, PL values were normalized for each minute of play, as per Montgomery et al. (2010) and Casamichana et al. (2012), and reported as the accumulated player workload per min (PLacc min $\left.{ }^{-1}\right)$.

\section{Procedures}

Specific training drills (Table 1) were chosen in accordance to previous research conducted by Rampinini et al. (2007) and Dellal et al. (2011). The number of games per format was as follows: 2 vs. 2 (SSG2), $\mathrm{n}=10 ; 3$ vs. 3 (SSG3), $\mathrm{n}=7 ; 4$ vs. 4 (SSG4), $\mathrm{n}=5$. Players were matched on technical ability and organized so that a player from each positional role was included. Games were possesion based and limited to two touches per player possession. Players were also analysed during six home English College friendly fixtures during the 2013-2014 season. All games were played on a full-size synthetic 3G surface; a 4-3-3 formation was preferred, and only players completing $90 \mathrm{~min}$ were included. Before the commencement of the SSGs and friendly games, a standardised 20 min warm up was completed.

Statistical Analysis

Data was first uploaded to proprietary software (Catapult, Sprint software, 5.0). A $4 \times 4$ mixed-model repeated measures ANOVA was used to determine significant main effects for condition and playing positions for each variable. Bonferroni post hoc analyses were then used to determine where differences lay. When a significant interaction was found, a one-way ANOVA was used to identify differences between positional roles in different conditions. Statistical significance was accepted at $p \leq 0.05$, while Eta squared $\left(\eta^{2}\right)$ was used to measure effect size, where $<0.2=$ small, $0.2-0.8=$ medium and $>0.8=$ large. All statistical procedures were completed using SPSS 20.0 (SPSS Inc. Chicago, USA).

\section{Results}

PLacc-min ${ }^{-1}$ was significantly higher in each SSG format compared to MP (SSG2: $15.00 \pm 3.53$; SSG3: $14.68 \pm 3.27$; SSG4: $13.47 \pm 3.35$ vs. MP: 10.18 $\pm 2.12 \mathrm{AU})$. $\mathrm{X}$ axis $\mathrm{PL} \cdot \mathrm{min}^{-1}$ was also significantly greater in each SSG format compared to MP (SSG2: $4.10 \pm 3.94$; SSG3: $3.92 \pm 0.89$; SSG4: $3.58 \pm$ 0.83 vs. MP: $2.61 \pm 0.54 \mathrm{AU})$. $\mathrm{Y}$ axis $\mathrm{PL} \cdot \mathrm{min}^{-1}$ was significantly greater in each SSG format compared to MP (SSG2: $4.00 \pm 1.29$; SSG3: $3.97 \pm 1.08$; SSG4: $3.74 \pm 1.16$ vs. MP: $2.66 \pm 0.75 \mathrm{AU})$, as was $\mathrm{Z}$ axis PL·min ${ }^{-1}$ (SSG2: $6.90 \pm 1.53$; SSG3: $6.80 \pm 1.55$; SSG4: $6.17 \pm 1.53$ vs. MP: $4.90 \pm 1.01$ AU (Figure 1).

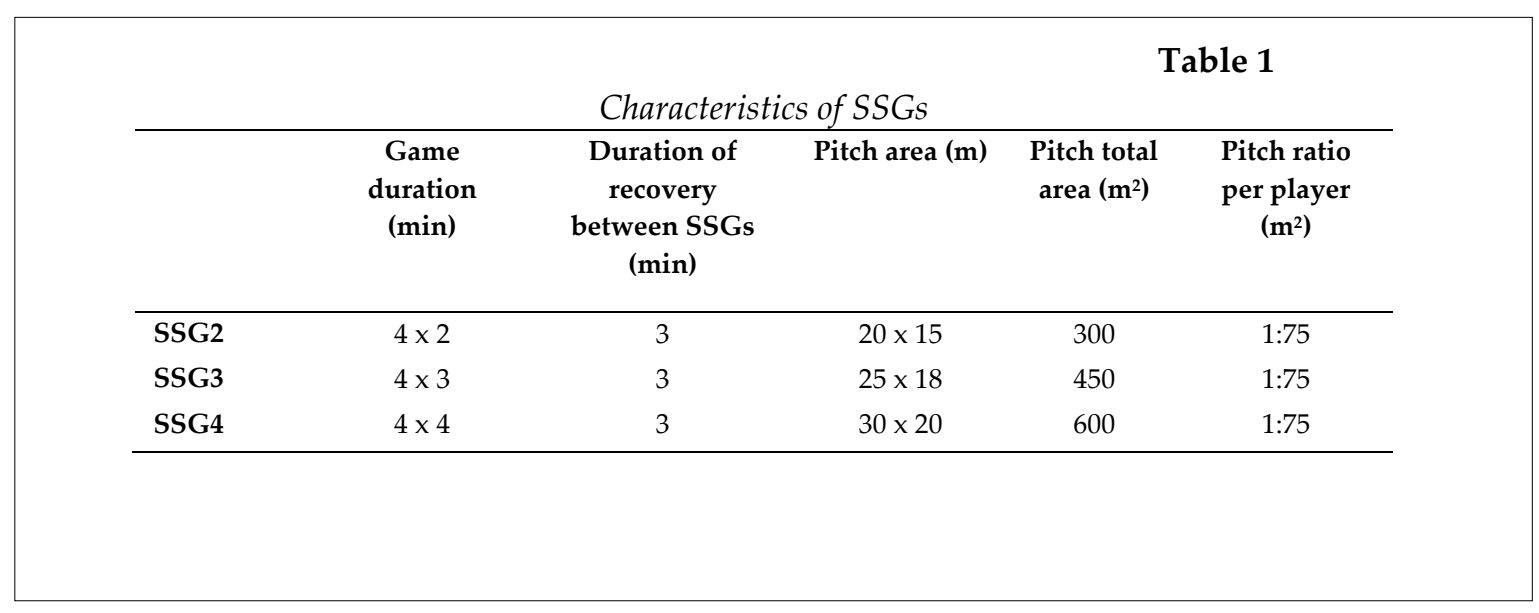



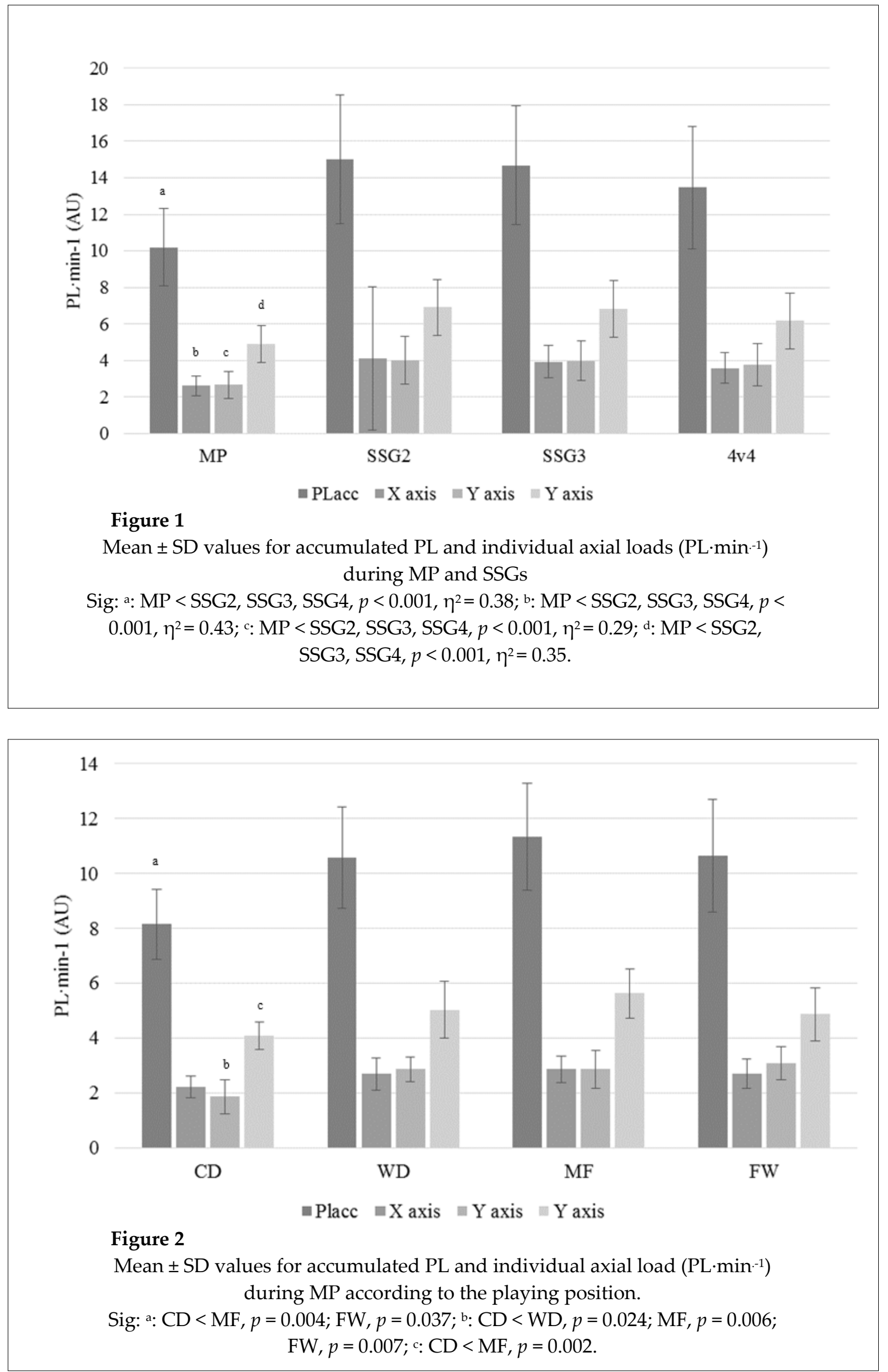
When mean PLace $\min ^{-1}$ was examined during MP, positional differences were identified. PLacc $\min ^{-1}$ was significantly greater for MF and FW than $\mathrm{CD}(11.34 \pm 1.95 ; 10.65 \pm 2.06$ vs. $8.15 \pm 1.28$ AU). A significant main effect was found for the positional role when the physical load in the $X$ axis per min was examined; however, post hoc tests revealed no significant differences between positional roles. When mean $\mathrm{Y}$ axis $\mathrm{PL} \cdot \mathrm{min}^{-1}$ was examined, a significant main effect was found for the positional role. In addition, WD, MF and FW were significantly greater than $\mathrm{CD}(2.86 \pm 0.45$; $2.87 \pm 0.69 ; 3.08 \pm 0.60$ vs. $1.86 \pm 0.62 \mathrm{AU}) . \mathrm{A}$ significant main effect was also found for $\mathrm{Z}$ axis $\mathrm{PL} \cdot \mathrm{min}^{-1}$ and MF was significantly greater than $\mathrm{CD}(5.63 \pm 0.90$ vs. $4.09 \pm 0.50 \mathrm{AU})$ (Figure 2).

\section{Discussion}

The main finding from this study was that PLacc. min $^{-1}$ was greater during each SSG modality than MP indicating that the external demands of SSGs were greater than MP. Research by Casamichana et al. (2012) is in agreement with the present study, in which PLacc. $\mathrm{min}^{-1}$ was significantly greater during SSGs $(15.8 \pm 2.7 \mathrm{AU})$ in comparison with MP (13.5 $\pm 1.5 \mathrm{AU})$. However, the current study provides more detailed information regarding the mechanical stress imposed on players of different positional roles through quantifying the discrete actions that occur during soccer.

During MP, MF reported the greatest PLacc $\min ^{-1}$ value, whereas CD reported the lowest values, consistent with the total PL during MP reported elsewhere (Barron et al., 2014). As the PL quantifies the total external load experienced by players, including the discrete, non-locomotor contributing activities such as jumping and collisions, these results provide further evidence that the position of MF indeed elicits greater work rate demands, with $C D$ requiring the least work rate (Di Salvo et al., 2007). A possible explanation for the greater PLacc $\cdot \mathrm{min}^{-1}$ reported by MF during MP could be due to the greater total distance covered by this position in comparison to others (Barron et al., 2014).

Across the SSG drills, an inverse relationship was observed between the number of players and external load, whereby formats with the fewest players elicited the greatest
PLacc. min $^{-1}$ values. A possible reason for this could be the reduction in technical demands as the number of player increase. The results of Aguiar et al. (2013) are in contrast to the findings of the present study, in which the 2 vs. 2 SSG format reported a PL value of $88.63 \pm 20.37 \mathrm{AU}$ that increased linearly along with an increase in the number of players with the 4 vs. 4 SSG format reporting a PL value of $95.18 \pm 17.54 \mathrm{AU}$. A possible reason for the discrepancies in these findings could be the different methodology employed compared to that of the present study. For example, the duration of the SSG2, SSG3, SSG4 modalities used in the present study was 8 , 12 and $16 \mathrm{~min}$, respectively, whereas the duration for all SSG in the study by Aguiar et al. (2013) was $18 \mathrm{~min}(6 \times 3 \mathrm{~min}$ bouts). It is expected that fatigue may have influenced the results in the aforementioned study as the ability to maintain high-intensity exercise would have been difficult in the later stages of the 2 vs. 2 SSG format. In contrast, during SSG3 and SSG4, players have a greater opportunity to recover allowing higherintensities to be sustained for a longer period. The ability to maintain higher intensity exercise during SSG3 and SSG4 in comparison to SSG2, is likely the reason explaining the increase in PLacc. $\mathrm{min}^{-1}$ as the number of players increase. Elsewhere, Gaudino et al. (2014) reported that moderate $\left(2-3 \mathrm{~m} \cdot \mathrm{s}^{-2}\right)$ and high accelerations $(>3$ $\mathrm{m} \cdot \mathrm{s}^{-2}$ ) were significantly more frequent in 5 vs. 5 and 7 vs. 7 compared to 10 vs. 10. A similar pattern was evident for moderate and high decelerations, supporting the findings of the present study that fewer players and smaller pitches elicit higher external loads.

The physical load in the $X$ axis was relatively homogenous across positional roles during MP. However, post hoc tests revealed that the mediolateral load was greater in all SSG formats compared to MP. Importantly, during SSGs the aim was to retain possession necessitating frequent changes of direction to evade opponents and find space to receive the ball. These discreet movements elicited a higher multi-directional load, consistent with the observations of Gaudino et al. (2014) unlike a goal-orientated focus that provides a direction for play characterized by more linear movement.

With regard to the physical load values in the $\mathrm{Y}$ axis, FW reported the greatest values with 
$\mathrm{CD}$ reporting the lowest values during MP, although the difference was not significant. A possible explanation for the differences could be that FW covered greater distances in acceleration / deceleration during speeding up and slowing down movements and / or rapid changes of direction. During MP, FW are obligated to move at high-speeds to evade defenders, whereas the $\mathrm{CD}$ has to track the FW movement and position himself strategically (Faude et al., 2012). Movement at these high intensities would likely result in anterior-posterior changes of the upper body position (i.e. forward and backward lean), and, therefore, a greater distance covered during high-intensity activities would increase the acceleration values in the $\mathrm{Y}$ axis. Cormack et al. (2013) observed an inverse relationship between anterior-posterior acceleration and high-speed running in a fatigued state and suggested that fewer anterior-posterior changes of the upper body position could indicate a less total distance covered in high-speed running. This is supported by the significantly greater $\mathrm{Y}$ axis values reported during all SSG than MP with values decreasing as the number of players increased. Furthermore, a greater distance covered at $>1 \mathrm{~m} \cdot \mathrm{s}^{-2}$ and $>-1 \mathrm{~m} \cdot \mathrm{s}^{-2}$ was reported for SSG2 and SSG3 in comparison to SSG4 suggesting a greater number of accelerations in the SSG and, therefore, greater changes in upper body positions.

Midfielders reported the greatest physical loads in the $\mathrm{Z}$ axis with $\mathrm{CD}$ reporting the lowest values during $\mathrm{MP}$, although the difference was not significant. Despite the fact that the data did not provide a definite conclusion to why MF reported the greatest values, it is possible that accelerations measured in the vertical plane reflected the PL accumulated from running and the associated vertical displacement. If, as previous work in netball, hockey and Australian football suggested, players that run at a higher intensity (including high-speed running and accelerating/decelerating during changes in velocity) involve more rapid vertical displacement than slower speed running, this could account for the greater contribution from the vertical vector (Brewer et al., 2010; Jennings et al., 2012).

This notion is supported by the significantly greater physical loads in the $\mathrm{Z}$ axis during SSG in comparisons to MP. Indeed, results from the present study demonstrated that although SSGs did not evoke high-speed running, they imposed a large physical demand on players through a greater accumulation of accelerations and decelerations. Cormack et al. (2013) found reductions in the Z-vector accelerometer values in the fatigued state, and, given that neuromuscular fatigue directly impairs the ability to sprint or accelerate / decelerate; this provides further support to the contribution of high-intensity activities such as acceleration / deceleration and sprinting to the Z-vector accelerometer. In order to improve player's capacity regarding match demands, an association of SSG and specific highintensity training is needed.

The matches and SSGs in this study were played on a $3 G$ surface, and to the best of our knowledge, the influence of the playing surface on PL has not been investigated. Greater muscular soreness has been reported following matches on artificial surfaces (Poulos et al., 2014) reflecting different surface properties (i.e.,.stiffness) (Williams et al., 2016) that may evoke higher running impact forces and greater PL. Given the increasing popularity of artificial surfaces, greater understanding about the relationship between PL and a playing surface may help inform player recovery strategies, particularly for those players unaccustomed to their use.

In conclusion, this study provides new insight into the differences in tri-axial PLacc $\mathrm{min}^{-1}$ during SSG and MP for different playing positions. Midfielders reported the greatest PL values during MP providing further support for the considerably greater work-rate demands of this positional role. SSG evoked considerably greater PLacc $\cdot \mathrm{min}^{-1}$ values for all positional roles in comparison to $\mathrm{MP}$, suggesting that previous time-motion analysis research using traditional constant-speed zones had underestimated the demands of soccer. Whilst the relative contribution of the $\mathrm{X}, \mathrm{Y}$ and $\mathrm{Z}$ axes to PL does not appear practically different between positional roles during MP, the greater values reported in SSG suggest that these games may provide a 'density' type-training stimulus, by imposing relative demands of acceleration and deceleration activities in excess of those experienced during MP. Coaches should therefore, carefully consider the scheduling of SSG, particularly in the lead up to competitive fixtures and during an early pre- 
season when players may not necessarily be conditioned to the high external workload demands.

\section{References}

Achten J, Jeukendrup AE. Heart rate monitoring: applications and limitations. Sports Med, 2003; 33: 517-538

Aguiar M, Bothelho G, Gonçalves B, Sampaio J. Physiological responses and activity profiles of football small-sided games. J Strength Cond Res, 2013; 27(5): 1287-1294

Barron DJ, Atkins S, Edmundson C, Fewtrell C. Accelerometer derived load according to playing position in competitive youth soccer. Int J Perf Anal Sport, 2014; 14: 734-743

Borresen J, Lambert M. Autonomic control of heart rate during and after exercise: measurements and implications for monitoring training status. Sports Med, 2008; 38: 633-646

Boyd L, Ball K, Aughey RJ. The reliability of MinimaxX accelerometers for measuring physical activity in Australian football. Int J Sports Physiol, 2011; 6: 311-321

Bradley P, Di Mascio M, Peart D, Olsen P, Sheldon B. High intensity activity profiles of elite soccer players at different performance levels. J Strength Cond Res, 2010; 111(6): 969-978

Brewer C, Dawson B, Heasman J, Stewart G, Cormack S. Movement pattern comparisons in elite (AFL) and sub-elite (WAFL) Australian football games using GPS. J Sci Med Sport, 2010; 13: 618-623

Buchheit M, Al Haddad H, Simpson B, Palazzi D, Bourdon P, Di Salvo V, Mendez-Villanueva A. Monitoring accelerations with GPS in football: time to slow down? Int J Sports Physiol Perform, 2014; 9(3): 442-446

Casamichana D, Castellano J, Castagna C. Comparing the physical demands of friendly matches and small sided games in semiprofessional soccer players. J Strength Cond Res, 2012; 26(3): 837-843

Clemente F, Martins F, Mendes R. Developing aerobic and anaerobic fitness using small-sided soccer games: methodological proposals. Strength Cond, 2014; 36: 76-87

Cormack S, Smith R, Mooney M, Young W, O'Brien B. Accelerometer load as a measure of activity profile in different standards of Netball match play. Int J Sports Physiol Perform, 2013; 9: 283-291

Cormack S, Mooney M, Morgan W, McGuigan M. Influence of neuromuscular fatigue on accelerometer load in elite Australian football players. Int J Sports Physiol Perform, 2013; 8: 373-378

Cummins C, Orr R, O'Connor H, West C. Global Positioning Systems (GPS) and microtechnology sensors in team sports: a systematic review. Sports Med, 2013; 43: 1025-1042

Dellal A, Chamari K, Pintus A, Girard O, Cotte T, Keller D. Heart rate responses during small-sided games and short intermittent running training in elite soccer players: a comparative study. J Strength Cond Res, 2008; 22(5): 1449-1457

Dellal A, Hill-Haas S, Lago-Penas C, Chamari K. Small-sided games in soccer: amateur vs. professional players' physiological responses, physical, and technical activities. J Strength Cond Res, 2011; 25(9): 2371-2381

Dellal A, Owen A, Wong D, Krustrup P, van Exsel M, Mallo J. Technical and physical demands of small vs. large sided games in relation to playing position in elite soccer. Hum Mov Sci, 2012; 31: 957-969

Di Salvo V, Baron R, Tschan H, Calderon Montero F, Bachl N, Pigozzi F. Performance characteristics according to playing position in elite soccer. Int J Sports Med, 2007; 28: 222-227

Edgecomb S, Norton K. Comparison of global positioning and computer-based tracking systems for measuring player movement distance during Australian football. J Sci Med Sport, 2006; 9: 25-32

Faude $\mathrm{O}$, Koch $\mathrm{T}$, Meyer T. Straight line sprinting is the most frequent action in goal situations in professional football. J Sports Sci, 2012; 30(7): 625-631 
Gabbett T. GPS analysis of elite women's field hockey training and competition. J Strength Cond Res, 2010; 24(5): 1321-1324

Gabbett T, Mulvey M. Time-motion analysis of small-sided training games and competition in elite women soccer players. J Strength Cond Res, 2008; 22(2): 543-552

Gaudino P, Giampietro A, Iaia M. Estimated metabolic and mechanical demands during different smallsided games in elite soccer players. Hum Mov Sci, 2014; 36: 123-133

Hill-Haas S, Dawson B, Impellizzeri F, Coutts A. Physiology of small-sided games training in football: a systematic review. Sports Med, 2011; 41: 199-220

Jennings D, Cormack S, Coutts AJ, Boyd L, Aughey R. The validity and reliability of GPS units for measuring distance in team sport specific running patterns. Int J Sports Physiol Perform, 2010; 5: 328-341

Jennings D, Cormack S, Coutts A, Boyd L, Aughey R. International field hockey players perform more highspeed running than national-level counterparts. J Strength Cond Res, 2012; 26(4): 947-952

Johnston R, Watsford M, Kelly S, Pine M, Spurris R. Validity and reliability of $10 \mathrm{~Hz}$ and $15 \mathrm{~Hz}$ GPS units for assessing athlete movement demands. J Strength Cond Res, 2014; 28(6): 1649-1655

Kelly D, Drust B. The effect of pitch dimensions on heart rate responses and technical demands of smallsided soccer games in elite players. J Sci Med Sport, 2009; 12: 475-479

Little T, Williams AG. Suitability of soccer training drills for endurance training. J Strength Cond Res, 2006; 20(2): 316-319

Little T, Williams AG. Measures of exercise intensity during soccer training drills with professional soccer players. J Strength Cond Res, 2007; 21(2): 367-371

Mallo J, Navarro E. Physical load imposed on soccer players during small-sided training games. J Sports Med Phys Fitness, 2008; 48: 166-171

Mohr M, Krustrup P, Bangsbo J. Match performance of high-standard players with special reference to development of fatigue. J Sports Sci, 2003; 21(7): 519-528

Montgomery P, Pyne D, Minahan C. The physical and physiological demands of basketball training and competition. Int J Sports Physiol Perform, 2010; 5: 75-86

Osgnach C, Poser S, Bernardini R, Rinaldo R, di Prampero P. Energy cost and metabolic power in elite soccer: a new match analysis approach. Med Sci Sport Exerc, 2010; 42: 170-178

Petersen C, Pyne D, Dawson B, Kellett A, Portus M. Comparison of training and game demands of national level cricketers. J Strength Cond Res, 2011; 25(5): 1306-1311

Poulos C, Gallucci J, Gage W, Baker J, Buitrage S, Macpherson A. The perceptions of professional soccer players on the risk of injury from competition and training on natural grass and $3^{\text {rd }}$ generation artificial turf. BMC Sports Sci Med Rehabil, 2014; 1(6): 11-18

Rampinini E, Coutts A, Castagna C, Sassi R, Impellizzeri F. Variation in top level soccer match performance. Int J Sports Med, 2007; 28: 1018-1024

Rampinini E, Impellizzeri F, Castagna C, Abt G, Chamari K, Sassi A, Marcora S. Factors influencing physiological responses to small-sided soccer games. J Sports Sci, 2007; 25: 659-666

Rawstorn JC, Maddison R, Ali A, Foskett A, Gant N. Rapid directional change degrades GPS distance measurement validity during intermittent intensity running. PLOS ONE, 2014; 9: 1-6

Reilly T, Bowen T. Exertional costs of changes in directional modes of running. Percep Mot Skills, 1984; 58: $149-150$

Scott B, Lockie R, Knight T, Clark A, Xanne A, Janse de Jonge X. A comparison of methods to quantify the inseason training load of professional soccer players. Int J Sports Physiol Perform, 2013; 8: 195-202

Thompson D, Nicholas C, Williams C. Muscular soreness following prolonged intermittent high-intensity 
shuttle running. J Sports Sci, 1999; 17(5): 387-395

Varley M, Aughey R. Acceleration profiles in elite Australian soccer. Int J Sports Med, 2013; 34(1): 34-39

Williams S, Trewartha G, Kemp S, Michell R, Stokes K. The influence of an artificial playing surface on perceptions of muscle soreness in elite Rugby Union. Scand J Med Sci Sports, 2016; 26: 101-108

\section{Corresponding author:}

\section{David Barron}

Centre for Research in Sports Performance, Myerscough College,

Bilsborrow, Preston, Lancashire, UK.

Phone: 01995642110

E-mail: dbarron@myerscough.ac.uk. 